\title{
Adolescent girl - the gordian knot
}

\author{
Lalitha S, Padmasri Ramalingappa
}

\author{
Correspondence: Dr Padmasri Ramalingappa, Prof and HOD, Dept of Obstetrics \& \\ Gynaecology, Sapthagiri Institute of Medical Sciences \& Research Centre, Chikkasandra, \\ Hesarghatta Main Road, Bangalore - 560090, Karnataka, India ; Email - \\ drpadmasuraj@gmail.com
}

Distributed under Attribution-NonCommercial-ShareAlike 4.0 International (CC BY-NC-SA 4.0)

\begin{abstract}
Objective: The aim of this study is to review the gynecological problems of the adolescent population attending the gynaecological outpatient department. Materials and Methods: The records of 75 adolescent girls in the age group 10-19 years attending the gynaecology outpatient department in a tertiary care hospital from July 2015 - December 2016 were analysed. Data regarding age at menarche, menstrual and marital history, history of gynaecological problems and other associated problems were also taken. General examination and clinical examination findings and lab reports such as hemogram, coagulogram, hormonal assay and pelvic ultrasound were analyzed from records. Results: Incidence of gynecological problem in our study was $6.25 \%$. Different gynecological problems were menstrual disorders ( $60 \%$ ), leucorrhoea ( $10.66 \%)$ ), infections ( $8 \%$ ), ovarian cyst ( $5.33 \%)$, sexual assault (2.66\%), teenage pregnancy $(10.66 \%)$ and infertility ( $2.66 \%)$. Majority of girls with menstrual disorders suffered from puberty menorrhagia (55.55\%). Abnormal uterine bleeding was diagnosed in $74.28 \%$ of girls suffering from puberty menorrhagia. Pelvic inflammatory disease was exclusively seen in married adolescent girls $(8 \%)$. Vaginal atresia and imperforate hymen were causes of primary amenorrhoea (4.44\%). PCOD was the commonest cause of secondary amenorrhoea amongst adolescents $(17.77 \%)$. Conclusion: Menstrual abnormalities are the most common problems of adolescent girls. Setting up adolescent clinics is desirable.
\end{abstract}

"No longer a child, not yet a women", is a line which captures the ethos of adolescence beautifully. This is a time of transition from childhood to becoming responsible adults. The changes are not only physical and sexual, but also emotional and mental. We, as health care providers, need to focus on these young people as investing in their health today will go a long way in boosting preventing health care in tomorrow's adults ${ }^{1}$. India has the largest population of adolescents in the world, accounts for a
Keywords: Adolescent, gynaecology, complications.

quarter of the country's population (243 million estimated - by UNICEF today in its 2011 state of the world's children report entitled 'Adolescence: An age of opportunity") ${ }^{2}$. WHO defines adolescents as individuals in the age group of 10-19 years $(2002-2003)^{3}$.

Gynaecological problems of adolescents occupy a special space in the spectrum of gynecological disorders of all ages. This is because of the physiological and psychological factors which are very important in the

Received: $7^{\text {th }}$ September 2018. Accepted: $5^{\text {th }}$ December 2018.

Lalitha S, Ramalingappa P. Adolescent girl - the gordian knot. The New Indian Journal of OBGYN. 2019; 5(2): 131-35 
growth and psychological remodeling of someone in the transition between childhood and womanhood. Yet this subspecialised area of gynaecology has still not been explored optimally ${ }^{4}$. Adolescent period is characterized by its own set of specific gynaecological problems like puberty and its disorders, menstrual irregularities, anemia, vaginal discharge, polycystic ovarian disease (PCOD), teenage pregnancy, pelvic tumors, pelvic inflammatory disease (PID), sexually transmitted disease (STD) and other obstetric problems. This big spectrum of problem remits a formidable challenge to the consultants in the field of obstetrics and gynecology. In this study, an attempt has been made to review the gynecological problems of the adolescent population attending the gynaecological outpatient of our hospital.

\section{Materials and Methods}

This cross-sectional study was done among adolescent girls from July 2015 - December 2016. The total numbers of patients attending the gynecological outpatient department, from July 2015 to December 2016, were recorded in the outpatient department (OPD) register. Adolescents in the age group of 11-19 years were identified. Those adolescent girls who had medical or surgical illness were excluded from the study. Records regarding their history, gynecological and other associated problems, general examination, height, weight and secondary sexual characteristics and investigation such as hemogram, coagulation profile, hormonal assay (follicle stimulating hormone, luetinising hormone and thyroid function test, prolactin) and pelvic ultrasound were analyzed. The results are expressed in tables as number and percentage.

\section{Results and Observations}

From the records, it was found that a total of 35,100

Table 1: Showing Age and BMI

\begin{tabular}{lll}
\hline Variables & & No $(\%)$ \\
\hline Age in & $<13$ & $7(9.33 \%)$ \\
years & $13-15$ & $10(13.33 \%)$ \\
& $15-17$ & $23(30.66 \%)$ \\
& $17-19$ & $35(46.66 \%)$ \\
\hline BMI & $<19$ & $52(69.33 \%)$ \\
(Body & $19-24.9$ & $15(20 \%)$ \\
Mass & $25-30$ & $8(10.66 \%)$ \\
Index) & $>30$ & - \\
\hline
\end{tabular}

attended the gynecology OPD during the study period. Out of them, $1200(3.41 \%)$ belonged to the adolescent age group, i.e. 11-19 years. Those adolescent girls who had

Table 2: Adolescent gynecological problems in outpatients

\begin{tabular}{ll}
\hline Types & Number (\%) \\
\hline Menstrual disorder & $45(60 \%)$ \\
Teenage pregnancy & $8(10.66 \%)$ \\
Leucorrhoea & $8(10.66 \%)$ \\
PID & $6(8 \%)$ \\
Ovarian cyst & $4(5.33 \%)$ \\
Sexual assault & $2(2.66 \%)$ \\
Infertility & $2(2.66 \%)$ \\
\hline
\end{tabular}

medical or surgical illness were excluded from the study. So, data from 75 adolescent girls who had gynecological problems were analysed. Maximum adolescent girls attending gynecological OPD had ages in the range of 1719 years i.e. $46.66 \%$ (Table -1). Majority of adolescent girls were underweight with BMI $<19$ (52 out of $75 ; 69.33$ $\%)$, (Table - 1). This could be due to poverty and illiteracy prevalent in our country.

Table 2 enumerates the various types of gynecological problems encountered in the study group. Menstrual disorders were the most common $(60 \%)$ problem for

Table 3: Menstrual disorder

\begin{tabular}{ll}
\hline Disorder & Number(\%) \\
\hline Puberty menorrhagia & $25(55.55 \%)$ \\
Dysmenorrhoea & $9(20 \%)$ \\
Oligomenorrhoea & $1(2.22 \%)$ \\
Primary Amenorrhoea & $2(4.44 \%)$ \\
Secondary Amenorrhoea & $8(17.77 \%)$ \\
\hline
\end{tabular}

them seeking professional advice. Teenage pregnancy was the second most common problem $(10.66 \%)$. Other conditions noted were leucorrhoea (10.66\%), PID (8\%), ovarian cyst/ tumors (5.33\%), sexual assault (2.66\%) and infertility (2.66\%).

Table 3 reveals that menstrual disorders varied from amenorrhoea in 10 cases $(13.3 \%)$ to menstrual dysfunction, which includes menorrhagia, metrorrhagia, dysmenorrhoea, oligo and hypomenorrhoea.

Puberty menorrhagia was present in 25 out of 45 (55.55\%), 9 out of 45 (20\%) cases had dysmenorrhoea, 1 
Table 4: Etiology of menstrual dysfunction, primary amenorrhoea, secondary amenorrhoea

\begin{tabular}{|c|c|c|}
\hline Variables & & No (\%) \\
\hline \multirow{5}{*}{$\begin{array}{l}\text { Menstrual dysfunction } \\
(\mathrm{N}=35)\end{array}$} & DUB & $26(74.28 \%)$ \\
\hline & PCOD & $6(17.14 \%)$ \\
\hline & OCP user and breakthough bleeding & $1(2.85 \%)$ \\
\hline & Thyroid disorder & $1(2.85 \%)$ \\
\hline & Marked psychological stress & $1(2.85 \%)$ \\
\hline \multirow{2}{*}{$\begin{array}{l}\text { Primary amenorrhoea } \\
(\mathrm{N}=2)\end{array}$} & Imperforated hymen & $1(50 \%)$ \\
\hline & Delayed puberty & $1(50 \%)$ \\
\hline \multirow{3}{*}{$\begin{array}{l}\text { Secondary amenorrhoea } \\
(\mathrm{N}=8)\end{array}$} & PCOD & $6(75 \%)$ \\
\hline & Hypothyroidism & $1(12.5 \%)$ \\
\hline & Tuberculosis of abdomen & $1(12.5 \%)$ \\
\hline
\end{tabular}

girls i.e. $10.66 \%$. Two married adolescent girls presented with infertility, sexual assault was reported in 2 out of $75(2.66 \%)$ adolescent girls.

\section{Discussion}

Adolescents present with myriads of gynecological problems. The most common among them are puberty menorrhagia, dysmenorrhoea and leucorrhoea. Proper counseling and sex education will make them aware of these problems.

In the present study, it was revealed that among the patients attending gynecology OPD, the adolescent age group patients with specific gynecological complaints were about $6.25 \%$. The less incidence in our hospital is because many of them are still shy and do not attend the regular gynecology out-patient department. So, special adolescent clinics will bring into light, many of the hidden adolescent problems.

The present work revealed that $46.66 \%$ of patients attending the gynecology OPD were in the late adolescent age group-17- 19 years. This study revealed that most of the adolescents were underweight $(69.33 \%)$. This condition is due to malnutrition and low-socioeconomic status.

Adolescent marriages are still prevalent in our state leading onto early sexual activity and teenage pregnancy and its complications. In the present study, $13.33 \%$ of adolescents are married, more prone for STDs and reproductive tract infections. Teenage pregnancies accounted for $4.03 \%$ of $(5 / 124)$ cases in the study conducted by Goswami Sebanti et $\mathrm{al}^{5}$ as compared to $10.66 \%$ in our study. In a study recently conducted in Banaras Hindu University Institute of Medical Science, teenage pregnancy was found to be associated with a significantly higher risk of pregnancy induced hypertension, preeclampsia and eclampsia, premature onset of labour, fetal death and premature delivery. Increased neonatal morbidity and mortality were also seen in babies delivered to teenage mothers.

In the present study, menstrual disorders were the most common gynaecological problem, comprising of 
about $60 \%$. Goswami Sebanti et al. conducted a study on 124 adolescent girls attending gynaecological OPD and concluded that menstrual disorders were the commonest gynecological problems $(58.06 \%)^{5}$. Another study conducted by Chand Karki et $\mathrm{al}^{6}$, revealed that menstrual disorders was seen in $23.80 \%$ of adolescent girls.

Menstrual disorders varied from menorrhagia ( 25 out of 45 ) in $55.55 \%$, dysmenorrhoea ( 9 out of 45 cases) in $20 \%$, oligomenorrhoea ( 1 out of 45 cases) in $2.22 \%$ and menorrhea (10 out of 45) in 22.25 of cases. In a similar data from one gynecological clinic at All India Institute of Medical Science (AIIMS) ${ }^{7}$, overall spectrum of problems among adolescent girls attending clinic were oligomenorrhoea (24\%), menorrhagia (16\%), dysmenorrhea $(12 \%)$, primary amenorrhoea $(12 \%)$, hirsutism (12\%), congenital anomalies of uterus//vagina $(12 \%)$ and urinary infections (12\%).

In the present study, dysfunctional uterine bleeding (DUB) was diagnosed in $74.28 \%$ of patients with abnormal uterine bleeding. No case with coagulation disorder was found. This study showed that though rare, leukemia and other coagulation disorders should be kept in mind while evaluating a case of puberty menorrhagia. A study conducted by Archana Kumari ${ }^{8}$, revealed that $60.2 \%$ of cases of menstrual irregularities were found to have DUB. Those patients with DUB who had anemia were treated with haematinics, antifibrinolytics and hormones (OCPs or progestogens).

The incidence of amenorrhoea among adolescent girls is $22.2 \%$, with secondary amenorrhoea being the most common $(80 \%)$ and primary amenorrhoea comprises about $20 \%$. Two out of 45 cases had primary amenorrhoea, the cause being imperforate hymen, which was treated with hymenectomy and the other case had constitutional delayed puberty and reassurance was given.

Of the 8 adolescent girls presenting with secondary amenorrhoea, 6 of them i.e. $75 \%$ had polycystic ovarian disease, $1(12.5 \%)$ had hypothyroidism and 1 had TB abdomen (12.5\%). Goswami Sebanti et $\mathrm{al}^{5}$, in their study found a similar result. In their study, most of the patients $(10 / 13)$ suffering from secondary amenorrhoea (duration of 4-5 months) or oligomenorrhoea were ultimately diagnosed to be cases of PCOD based of clinical criteria of menstrual problems, features of hyperandrogenism and sonography findings. Bhattacharya and Jha $(2014)^{9}$ studied 96 adolescent girls, 51 (53.13\%) of them had PCOS. Childhood obesity is associated with early onset of puberty, menstrual irregularities and polycystic ovarian syndrome during adolescence ${ }^{10}$.

In the present study, leucorrhoea was found in 8 out of 75 girls, i.e. $10.66 \%$.Leucorrhoea is a frequent and embarrassing problem, especially in low socio-economic population. In this study, 6 out of 75 i.e. $8 \%$ of patients were presented with infection. Pelvic inflammatory disease was seen exclusively in married adolescent girls owing to early sexual activity. Physiological cyst of ovary was found in 3 out of 75 adolescent girls and 1 patient had ovarian tumour (mixed germ cell tumour). A study by Samarth $\mathrm{S}$ et $\mathrm{al}^{11}$ revealed that benign ovarian cysts were found in $6(1.86 \%)$ out of 321 and none of them had malignant ovarian disease. Sexual assault was seen in 2 out of 75 (2.66 \%) of adolescent girls in our study. Sexual assault may lead to lower self-esteem, depression, unwanted pregnancies and criminal abortion amongst adolescents. These girls need to be counseled so that they do not end up having unwanted pregnancy and criminal abortion.

\section{Conclusion}

Menstrual disorders were the commonest gynecological problem of adolescents. The commonest among them are menorrhagia, dysmenorrhoea and leucorrhoea and their misbeliefs were alleviated, by giving them basis knowledge about the anatomy and physiology of human reproductive system and reassurance. Maintenance of menstrual hygiene is very important for a girl to protect herself from infections. But it is equally important for girls to have a feeling of wellbeing even during periods and not see them as a monthly punishment or sickness.

\section{Conflict of interest: None. Disclaimer: Nil.}

\section{References}

1.UNFPA. The state of the world population, 2003. Making one billion counts: investing in adolescents' health rights. New York: UNFPA; 2006.

2.Jain RB. Kumar A, Khanna P. Assessment of selfawareness among rural adolescents. A cross-sectional study. Indian Journal of Endocrinology and Metabolism. 2013; 17 (17): 367-72. 
3.Department of health service. National adolescent Health and Development strategy. Kathmandu; Ministry of Health, His majesty's Government Nepal; 2000.

4.Goswami P, Ahirncu G, Mishra P, Agarwal V. Adolescent Gynecological problems: A Prospective study. Journal of Evolution of medical and Dental Sciences. 2015; 4(102): 16709-12.

5.Goswami S, Dutta R, Sengupta S. A Profile of adolescent girls with gynecological problems. Journal of Obstetrics and Gynecology of India. 2005; 55(4) : 353-5.

6.Karki C, Singh Shrestha N, Rayamajni RT. Gynecological disorders of adolescent girls at Kathmandu medical college teaching Hospital. NJOG. 2008; 3 (2): 44-7.

7.Kumar S. Adolescent gynecological Problems. Obstet Gynaecol Today. 2000; V ( 6 ): 353-64.

8.Kumari A. Adolescent Gynecological Problems: A clinical Study. Journal of Evolution of medical and Dental Sciences. 2013; V (2): 1111-5.
9. Bhattacharya SM, Jha A. Prevalence and risk of metabolic syndrome in adolescent Indian girls with polycystic ovary syndrome using the 2009 - joint interim criteria. J Obstet Gynaecol Res. 2011; 37(10): 1303-7.

10.Franks S. Polycystic ovary syndrome in adolescents. Int $\mathrm{J}$ Obes (Lond). 2008; 32:1035-41.

11. Samarth S, Inandar S, Hariharan C, Kalyani KR. Study of various gynaecological problems and reproductive health awareness amongst adolescents at a rural setup in central India. Int J Reprod Contracept Obstet Gynecol. 2014; 3 : 1010-4.

Lalitha $\mathbf{S}^{\mathbf{1}}$, Padmasri Ramalingappa
${ }^{1}$ Assistant Professor; ${ }^{2}$ Professor and Head of
Department, Dept of Obstetrics \& Gynaecology,
Sapthagiri Institute of Medical Sciences \& Research
Centre, Chikkasandra, Hesarghatta Main Road,
Bangalore - 560090, Karnataka, India.

